\title{
Reinforcement Learning in Nonzero-sum Linear Quadratic Deep Structured Games: Global Convergence of Policy Optimization
}

\author{
Masoud Roudneshin, Jalal Arabneydi and Amir G. Aghdam
}

\begin{abstract}
We study model-based and model-free policy optimization in a class of nonzero-sum stochastic dynamic games called linear quadratic (LQ) deep structured games. In such games, players interact with each other through a set of weighted averages (linear regressions) of the states and actions. In this paper, we focus our attention to homogeneous weights; however, for the special case of infinite population, the obtained results extend to asymptotically vanishing weights wherein the players learn the sequential weighted mean-field equilibrium. Despite the non-convexity of the optimization in policy space and the fact that policy optimization does not generally converge in game setting, we prove that the proposed model-based and model-free policy gradient descent and natural policy gradient descent algorithms globally converge to the sub-game perfect Nash equilibrium. To the best of our knowledge, this is the first result that provides a global convergence proof of policy optimization in a nonzero-sum LQ game. One of the salient features of the proposed algorithms is that their parameter space is independent of the number of players, and when the dimension of state space is significantly larger than that of the action space, they provide a more efficient way of computation compared to those algorithms that plan and learn in the action space. Finally, some simulations are provided to numerically verify the obtained theoretical results.
\end{abstract}

\section{INTRODUCTION}

In recent years, there has been a growing interest in the application of reinforcement learning (RL) algorithms in networked control systems. One of the most popular reinforcement learning (RL) algorithms in practice is policy gradient, due to its stability and fast convergence. However, from the theoretical point of view, there is not much known about it. Recently, it is shown in [1] that a single-agent linear quadratic (LQ) optimal control problem enjoys the global convergence, despite the fact that the optimization problem is not convex in the policy space. A similar result is obtained for zero-sum LQ games in [2]. On the other hand, a nonzero-sum LQ game is more challenging than the above problems, where the existing results on the global (or even local) convergence of the policy gradient methods are generally not encouraging [3].

Inspired by recent developments in deep structured teams and games [4], [5], [6], [7], [8], [9], we study a class of LQ games wherein the effect of other players on any individual player is characterized by a linear regression of the states and actions of all players. The closest field of research to deep

This work is supported in part by the Natural Sciences and Engineering Research Council of Canada (NSERC) under Grant RGPIN-262127-17.

Masoud Roudneshin, Jalal Arabneydi, and Amir G. Aghdam are with the Department of Electrical and Computer Engineering, Concordia University, 1455 de Maisonneuve Blvd. West, Montreal, QC, Canada, Postal Code: H3G 1M8. Email: m_roundnedencs.concordia.ca, jalal.arabneydi@mail.mcgill.ca,

aghdameece.concordia.ca structured games is mean-field games [10]. In a classical LQ mean-field game, one often has: (a) homogeneous individual weights (i.e., players are equally important); (b) the number of players $n$ is asymptotically large with independent primitive random variables (to be able to predict the trajectory of the mean-field using the strong law of large numbers); (c) the coupling is through the mean of the states, where the control coupling (called extended coupling) is more challenging; (d) the proof technique revolves around the fact that the effect of a single player on others is negligible, reducing the game to a coupled forward-backward optimal control problem; (e) the solution concept is Nash equilibrium; (f) given some fixed-point conditions across the time horizon, the forwardbackward equation admits a solution leading to an approximate Nash in the finite-population game; $(\mathrm{g})$ they are often not practical for long-horizon and reinforcement learning applications wherein the common practice is to adopt a weaker solution concept called stationary Nash equilibrium (where the trajectory of the mean-field is stationary), and (h) since the results are asymptotic, the models are limited to those that are uniformly bounded in $n$. In contrast to mean-field game, LQ deep structured game often has: (a') heterogeneous individual weights that are not necessarily homogeneous; (b') the number of players is arbitrary (not necessarily very large) with possibly correlated primitive random variables; (c') the coupling is through the weighted mean of the states and actions; (d') the proof technique revolves around a gauge transformation initially proposed in [11] (not based on the negligible effect); (e') the solution concept is sequential Nash; (f') the solution is exact (not an approximate one) for any arbitrary number of players and it is identified by Riccati equations; (g') since the solution concept is sequential, it is well suited for long-horizon and reinforcement learning, and (h') since the results are also valid for finite-population game, the dynamics and cost are not necessarily limited to uniformly bounded functions with respect to $n$. It is shown in [5] that the classical LQ mean-field game with the tracking cost formulation is a special case of deep structured games under standard conditions, where the meanfield equilibrium coincides with the sequential mean-field equilibrium. It is to be noted that the LQ mean-field-type game [12], [13], [14] is a single-agent control problem (i.e., it is not a non-cooperative game), which resembles a team problem with social welfare cost function 1 In particular, it may be viewed as a special case of risk-neutral LQ mean-

\footnotetext{
${ }^{1}$ When the mean field is replaced by the expectation of the state of the genetic player, the resultant problem is called mean-field-type game.
} 
field teams introduced in [11], showcased in [15], [16], [17], [18], [19], and extended to deep structured LQ teams in [6]. The interested reader is referred to [5, Section VI] for more details on similarities and differences between mean-field games, mean-field-type games and mean-field teams.

The rest of the paper is organized as follows. In Section II. the problem of LQ deep structured game is formulated. In Section III. the global convergence of model-based and model-free policy gradient descent and natural policy gradient descent algorithms are presented. In Section IV, some numerical examples are provided to validate the theoretical results. The paper is concluded in Section $\mathrm{V}$

\section{PROBlem Formulation}

Throughout the paper, $\mathbb{R}, \mathbb{R}_{>0}$ and $\mathbb{N}$ refer to the sets of real, positive real and natural numbers, respectively. Given any $n \in \mathbb{N}, \mathbb{N}_{n}, x_{1: n}$ and $\mathbf{I}_{n \times n}$ denote the finite set $\{1, \ldots, n\}$, vector $\left(x_{1}, \ldots, x_{n}\right)$ and the $n \times n$ identity matrix, respectively. $\|\cdot\|$ is the spectral norm of a matrix, $\|\cdot\|_{F}$ is the Frobenius norm of a matrix, $\operatorname{Tr}(\cdot)$ is the trace of a matrix, $\sigma_{\min }(\cdot)$ is the minimum singular value of a matrix, $\rho(\cdot)$ is the spectral radius of a matrix, and $\operatorname{diag}\left(\Lambda_{1}, \Lambda_{2}\right)$ is the block diagonal matrix $\left[\begin{array}{lll}\Lambda_{1} & 0 ; 0 & \Lambda_{2}\end{array}\right]$. For vectors $x, y$ and $z, \operatorname{vec}(x, y, z)=\left[x^{\top}, y^{\top}, z^{\top}\right]^{\top}$ is a column vector. The superscript $-i$ refers to all players except the $i$-th player. In addition, poly $(\cdot)$ denotes polynomial function.

Consider a nonzero-sum stochastic dynamic game with $n \in \mathbb{N}$ players. Let $x_{t}^{i} \in \mathbb{R}^{d_{x}}, u_{t}^{i} \in \mathbb{R}^{d_{u}}$ and $w_{t}^{i} \in \mathbb{R}^{d_{x}}$ denote the state, action and local noise of player $i \in \mathbb{N}_{n}$ at time $t \in \mathbb{N}$, where $d_{x}, d_{u} \in \mathbb{N}$. Define the weighted averages:

$$
\bar{x}_{t}:=\sum_{i=1}^{n} \alpha_{n}^{i} x_{t}^{i}, \quad \bar{u}_{t}:=\sum_{i=1}^{n} \alpha_{n}^{i} u_{t}^{i},
$$

where $\alpha_{n}^{i} \in \mathbb{R}$ is the influence factor (weight) of player $i$ among its peers. From [4], [5], [6], we refer to the above linear regressions as deep state and deep action in the sequel. To ease the presentation, the weights are normalized as follows: $\sum_{i=1}^{n} \alpha_{n}^{i}=1$.

The initial states $\left\{x_{1}^{1}, \ldots, x_{1}^{n}\right\}$ are random with finite covariance matrices. The evolution of the state of player $i \in \mathbb{N}_{n}$ at time $t \in \mathbb{N}$ is given by:

$$
x_{t+1}^{i}=A x_{t}^{i}+B u_{t}^{i}+\bar{A} \bar{x}_{t}+\bar{B} \bar{u}_{t}+w_{t}^{i},
$$

where $\left\{w_{t}^{i}\right\}_{t=1}^{\infty}$ is an i.i.d. zero-mean noise process with a finite covariance matrix. The primitive random variables $\left\{\left\{x_{1}^{i}\right\}_{i=1}^{n},\left\{w_{1}^{i}\right\}_{i=1}^{n},\left\{w_{2}^{i}\right\}_{i=1}^{n}, \ldots\right\}$ are defined on a common probability space and are mutually independent across time. The above random variables can be non-Gaussian and correlated (not necessarily independent) across players. The cost of player $i \in \mathbb{N}_{n}$ at time $t \in \mathbb{N}$ is given by:

$$
\begin{aligned}
c_{t}^{i}= & \left(x_{t}^{i}\right)^{\top} Q x_{t}^{i}+2\left(x_{t}^{i}\right)^{\top} S^{x} \bar{x}_{t}+\left(\bar{x}_{t}\right)^{\top} \bar{Q} \bar{x}_{t} \\
& +\left(u_{t}^{i}\right)^{\top} R u_{t}^{i}+2\left(u_{t}^{i}\right)^{\top} S^{u} \bar{u}_{t}+\left(\bar{u}_{t}\right)^{\top} \bar{R} \bar{u}_{t},
\end{aligned}
$$

where $Q, S^{x}, \bar{Q}, R, S^{u}$ and $\bar{R}$ are symmetric matrices with appropriate dimensions.
From [4], [5], [6], an information structure called deep state sharing (DSS) is considered wherein each player $i \in \mathbb{N}_{n}$ at any time $t \in \mathbb{N}$ observes its local state $x_{t}^{i}$ and the deep state $\bar{x}_{t}$, i.e., $u_{t}^{i}=g_{t}^{i}\left(x_{1: t}^{i}, \bar{x}_{1: t}\right)$, where $g_{t}^{i}$ is a measurable function adapted to the filteration of the underlying primitive random variables of $\left\{x_{1: t}^{i}, \bar{x}_{1: t}\right\}$. When the number of players is very large, one can use no-sharing (NS) information structure wherein each player observes only its local state. However, such a fully decentralized information structure comes at a price that one must predict the trajectory of the deep state in time (which introduces the computational complexity in time horizon in terms of storage and computation). For example, if the dynamics of the deep state (i.e., $A+\bar{A}$ and $B+\bar{B}$ ) is known (which is not applicable for model-free applications), the deep state can be predicted a head of time when primitive random variables are mutually independent by the strong law of large numbers. Alternatively, one can assume to have access to an external simulator for the dynamics of the deep state (which is basically DSS structure). In this paper, we focus on DSS information structure wherein there is no loss of optimality in restricting attention to stationary strategies despite the fact that the deep state is not stationary. The interested reader is referred to [5] for the convergence analysis of NS (approximate) solution to the DSS solution, as $n \rightarrow \infty$.

Define $\mathbf{g}_{n}^{i}:=\left\{g_{t}^{i}\right\}_{t=1}^{\infty}$ and $\mathbf{g}_{n}:=\left\{\mathbf{g}^{1}, \ldots, \mathbf{g}^{n}\right\}$. The admissible set of actions are square integrable such that $\mathbb{E}\left[\sum_{t=1}^{\infty} \gamma^{t-1}\left(u_{t}^{i}\right)^{\top} u_{t}^{i}\right]<\infty$. Given a discount factor $\gamma \in$ $(0,1)$, the cost-to-go for any player $i \in \mathbb{N}_{n}$ is described by:

$$
J_{n, \gamma}^{i}\left(\mathbf{g}_{n}^{i}, \mathbf{g}_{n}^{-i}\right)_{t_{0}}=(1-\gamma) \mathbb{E}\left[\sum_{t=t_{0}}^{\infty} \gamma^{t-1} c_{t}^{i}\right], \quad t_{0} \in \mathbb{N} .
$$

Problem 1. Suppose that the weights are homogeneous, i.e. $\alpha_{n}^{i}=\frac{1}{n}, i \in \mathbb{N}_{n}$. When a sequential Nash strategy $\mathbf{g}_{n}^{*}$ exists, develop model-based and model-free gradient descent and natural policy gradient descent procedures under DSS information structure such that for any player $i \in \mathbb{N}_{n}$ at any stage of the game $t_{0} \in \mathbb{N}$, and any arbitrary strategy $\mathbf{g}^{i}$ :

$$
J_{n, \gamma}^{i}\left(\mathbf{g}_{n}^{*, i}, \mathbf{g}_{n}^{*,-i}\right)_{t_{0}} \leq J_{n, \gamma}^{i}\left(\mathbf{g}^{i}, \mathbf{g}_{n}^{*,-i}\right)_{t_{0}} .
$$

Remark 1. It is to be noted that Problem 1 holds for arbitrary number of players $n$, where the solution depends on $n$. Since the infinite-population solution is easier for analysis and may be viewed as a special case, one can generalize the homogeneous weights $\alpha_{n}^{i}=\frac{1}{n}$ to heterogeneous weights $\alpha_{n}^{i}=\frac{\beta^{i}}{n}$, where $\beta^{i} \in\left[-\beta_{\max }, \beta_{\max }\right], \beta_{\max } \in \mathbb{R}_{>0}, i \in \mathbb{N}_{n}$. The resultant solution is called sequential weighted meanfield equilibrium (SWMFE) in [5]. The SWMFE constructs an approximate solution at any stage of the game $t_{0} \in \mathbb{N}$ such that $J_{n, \gamma}^{i}\left(\mathbf{g}_{\infty}^{*, i}, \mathbf{g}_{\infty}^{*,-i}\right)_{t_{0}} \leq J_{n, \gamma}^{i}\left(\mathbf{g}^{i}, \mathbf{g}_{\infty}^{*,-i}\right)_{t_{0}}+\varepsilon(n)$, where $\lim _{n \rightarrow \infty} \varepsilon(n)=0$. For more details, see [5, Theorem 4].

\section{A. Main challenges and contributions}

There are several challenges to solve Problem 11. The first one is the curse of dimensionality, where the computational complexity of the solution increases with the number 
of players. The second one is the imperfect information structure, where players do not have perfect information about the states of other players. The third challenge is that the resultant optimization problem is non-convex in the policy space, see a counterexample in [1]. The forth one lies in the fact that policy optimization is not even locally convergent in a game with continuous spaces, in general; see a counterexample in [3]. The main contribution of this paper is to present an analytical proof for the global convergence of model-based and model-free policy gradient algorithms. In contrast to the model-based solution in [5] (whose number of unknowns increases quadratically with $d_{x}$ ), the number of unknown parameters in the proposed algorithms increases linearly with $d_{x}$ and $d_{u}$. To the best of our knowledge, this is the first result on the global convergence of policy optimization in nonzero-sum LQ games.

\section{MAIN RESULTS}

In this section, we first present a model-based algorithm introduced in [5] that requires $2 d_{x} \times 2 d_{x}$ parameters to construct the solution. Then, we propose two model-based gradient algorithms and prove their global convergence to the above solution, where their planning space is the policy space (that requires $2 d_{u} \times d_{x}$ parameters to identify the solution). Based on the proposed gradient methods, we develop two model-free (reinforcement learning) algorithms and establish their global convergence to the model-based solution.

From [5], we use a gauge transformation to define the following variables for any player $i \in \mathbb{N}_{n}$ at any time $t \in \mathbb{N}$ : $\mathbf{x}_{t}^{i}:=\operatorname{vec}\left(x_{t}^{i}-\bar{x}_{t}, \bar{x}_{t}\right), \mathbf{u}_{t}^{i}:=\operatorname{vec}\left(u_{t}^{i}-\bar{u}_{t}, \bar{u}_{t}\right)$ and $\mathbf{w}_{t}^{i}:=$ $\operatorname{vec}\left(w_{t}^{i}-\bar{w}_{t}, \bar{w}_{t}\right)$, where $\bar{w}_{t}:=\frac{1}{n} \sum_{i=1}^{n} w_{t}^{i}$. In addition, we define the following matrices: $\mathbf{A}:=\operatorname{diag}(A, A+\bar{A}), \mathbf{B}:=$ $\operatorname{diag}(B, B+\bar{B})$, and

$\mathbf{Q}:=\left[\begin{array}{cc}Q & Q+S^{x} \\ Q+S^{x} & Q+2 S^{x}+\bar{Q}\end{array}\right], \quad \mathbf{R}:=\left[\begin{array}{cc}R & R+S^{u} \\ R+S^{u} & R+2 S^{u}+\bar{R}\end{array}\right]$.

We now express the per-step cost of each player in (1) as:

$$
c_{t}^{i}=\left(\mathbf{x}_{t}^{i}\right)^{\top} \mathbf{Q} \mathbf{x}_{t}^{i}+\left(\mathbf{u}_{t}^{i}\right)^{\top} \mathbf{R} \mathbf{u}_{t}^{i} .
$$

To formulate the solution, we present a non-standard algebraic Riccati equation, introduced in [5], as follows:

$$
\mathbf{M}(\boldsymbol{\theta})=\mathbf{Q}+\boldsymbol{\theta}^{\boldsymbol{\top}} \mathbf{R} \boldsymbol{\theta}+\gamma(\mathbf{A}-\mathbf{B} \boldsymbol{\theta})^{\top} \mathbf{M}(\boldsymbol{\theta})(\mathbf{A}-\mathbf{B} \boldsymbol{\theta}),
$$

where $\boldsymbol{\theta}:=\operatorname{diag}(\theta(n), \bar{\theta}(n)), \theta(n):=\left(F_{n}\right)^{-1} K_{n}, \bar{\theta}(n):=$ $\left(\bar{F}_{n}\right)^{-1} \bar{K}_{n}$, and matrices $F_{n}, \bar{F}_{n}, K_{n}$ and $\bar{K}_{n}$ are given by:

$$
\begin{aligned}
F_{n} & =\left(1-\frac{1}{n}\right)\left[R+\gamma B^{\top} \mathbf{M}^{1,1}(\boldsymbol{\theta}) B\right] \\
+ & \frac{1}{n}\left[R+S^{u}+\gamma(B+\bar{B})^{\top} \mathbf{M}^{1,2}(\boldsymbol{\theta}) B\right], \\
\bar{F}_{n} & =\left(1-\frac{1}{n}\right)\left[R+S^{u}+\gamma B^{\top} \mathbf{M}^{2,1}(\boldsymbol{\theta})(B+\bar{B})\right] \\
+ & \frac{1}{n}\left[R+2 S^{u}+\bar{R}+\gamma(B+\bar{B})^{\top} \mathbf{M}^{2,2}(\boldsymbol{\theta})(B+\bar{B})\right], \\
K_{n} & =\left(1-\frac{1}{n}\right) \gamma B^{\top} \mathbf{M}^{1,1}(\boldsymbol{\theta}) A+\frac{\gamma}{n}(B+\bar{B})^{\top} \mathbf{M}^{1,2}(\boldsymbol{\theta}) A, \\
\bar{K}_{n} & =\left(1-\frac{1}{n}\right) \gamma B^{\top} \mathbf{M}^{2,1}(\boldsymbol{\theta})(A+\bar{A})+\frac{\gamma}{n}(B+\bar{B})^{\top} \mathbf{M}^{2,2}(\boldsymbol{\theta})(A+\bar{A})
\end{aligned}
$$

Assumption 1. Suppose equations (4) and (5) admit a unique stable solution, which is also the limit of the finitehorizon solution. In addition, let $F_{n}$ and $\bar{F}_{n}$ be invertible matrices, and $\left(1-\frac{1}{n}\right) F_{n}+\frac{1}{n} \bar{F}_{n}$ be a positive definite matrix.

We now provide two sufficient conditions for Assumption 1 ensuring the existence of a stationary solution. Let $G$ denote the mapping from $\mathbf{M}$ to $\boldsymbol{\theta}$ displayed in (5) (where $\boldsymbol{\theta}=G(\mathbf{M})$ ), and $L$ denote the mapping from $\boldsymbol{\theta}$ to $\mathbf{M}$ expressed in (4) (where $\mathbf{M}=L(\boldsymbol{\theta})$ ). Thus, $\mathbf{M}=L(G(\mathbf{M})$ ) is a fixed-point equation to be solved by fixed-point methods.

Assumption 2. Let the mapping $L(G(\cdot))$ be a contraction, implying that equations (4) and (5) admit a unique fixedpoint solution. In addition, let $F_{n}$ and $\bar{F}_{n}$ be invertible matrices, and $\left(1-\frac{1}{n}\right) F_{n}+\frac{1}{n} \bar{F}_{n}$ be a positive definite matrix.

Assumption 3 (Infinite-population decoupled Riccati equations). Let $Q$ and $Q+S^{x}$ be positive semi-definite, $R$ and $R+S^{u}$ be positive definite, and $\bar{A}$ and $\bar{B}$ be zero. Suppose $(A, B)$ is stabilizable, and $\left(A, Q^{1 / 2}\right)$ and $\left(A,\left(Q+S^{x}\right)^{1 / 2}\right)$ are detectable. When $n$ is asymptotically large, the nonstandard Riccati equation (4) decomposes into two decoupled standard Riccati equations; see [5, Proposition 2].

Theorem 1 (Model-based solution using non-standard Riccati equation [5]). Let Assumption 1 hold. There exists a stationary subgame perfect Nash equilibrium such that for any player $i \in \mathbb{N}_{n}$ at any time $t \in \mathbb{N}_{T}$,

$$
u_{t}^{*, i}=-\theta^{*}(n) x_{t}^{i}-\left(\bar{\theta}^{*}(n)-\theta^{*}(n)\right) \bar{x}_{t},
$$

where the gains are obtained from (5). In addition, the optimal cost of player $i \in \mathbb{N}_{n}$ from the initial time $t_{0}=1$ is given by: $J_{n, \gamma}^{i}\left(\boldsymbol{\theta}^{*}\right)=(1-\gamma) \operatorname{Tr}\left(\mathbf{M}\left(\boldsymbol{\theta}^{*}\right) \boldsymbol{\Sigma}_{x}^{i}\right)+\gamma \operatorname{Tr}\left(\mathbf{M}\left(\boldsymbol{\theta}^{*}\right) \boldsymbol{\Sigma}_{w}^{i}\right)$, where $\boldsymbol{\Sigma}_{x}^{i}:=\mathbb{E}\left[\left(\operatorname{vec}\left(\Delta x_{1}^{i}\right), \bar{x}_{1}\right)\left(\operatorname{vec}\left(\Delta x_{1}^{i}\right), \bar{x}_{1}\right)^{\top}\right]$ and $\left.\left.\boldsymbol{\Sigma}_{w}^{i}:=\mathbb{E}\left[\operatorname{vec}\left(\Delta w_{t}^{i}\right), \bar{w}_{t}\right) \operatorname{vec}\left(\Delta w_{t}^{i}\right), \bar{w}_{t}\right)^{\top}\right]$.

\section{A. Model-based solution using policy optimization}

From Theorem 1, there is no loss of optimality in restricting attention to linear identical stationary strategies of the form $\boldsymbol{\theta}=\operatorname{diag}(\theta, \bar{\theta})$. Therefore, we select one arbitrary player $i$ as a learner and other players as imitators (that are passive during the learning process). More precisely, at each time instant, player $i$ uses a gradient algorithm to update its strategy whereas other players employ the updated strategy to determine their next actions. In this article, we discard the process of selecting the learner, but in order to have a fair implementation, the learner may be chosen randomly at each iteration 2 For simplicity of presentation, we omit the superscript $i$ and the subscription of the cost function. Hence, the strategy of the learner can be described by: $\mathbf{u}_{t}=-\boldsymbol{\theta} \mathbf{x}_{t}, \mathbf{u}_{t} \in \mathbb{R}^{2 d_{u}}, \mathbf{x}_{t} \in \mathbb{R}^{2 d_{x}}, t \in \mathbb{N}$.

Lemma 1. The following holds at the initial time $t_{0}=1$ :

$$
\left[\nabla_{\theta} J(\boldsymbol{\theta}), \nabla_{\bar{\theta}} J(\boldsymbol{\theta})\right]=2 \mathbf{P}_{n} \mathbf{E}_{\boldsymbol{\theta}} \boldsymbol{\Sigma}_{\boldsymbol{\theta}}
$$

\footnotetext{
${ }^{2}$ For the special case of infinite population, it is also possible that all players become learners, i.e., they simultaneously learn the strategies as long as their exploration noises are i.i.d. In such a case, the infinite-population deep state reduces to weighted mean-field and remains unchanged.
} 
where

$$
\left\{\begin{array}{l}
\mathbf{P}_{n}:=\left[\left(1-\frac{1}{n}\right) \mathbf{I}_{d_{u} \times d_{u}}, \frac{1}{n} \mathbf{I}_{d_{u} \times d_{u}}\right] \\
\mathbf{E}_{\boldsymbol{\theta}}:=\left(\mathbf{R}+\gamma \mathbf{B}^{\top} \mathbf{M}(\boldsymbol{\theta}) \mathbf{B}\right) \boldsymbol{\theta}-\gamma \mathbf{B}^{\top} \mathbf{M}(\boldsymbol{\theta}) \mathbf{A} \\
\boldsymbol{\Sigma}_{\boldsymbol{\theta}}:=\mathbb{E}\left[(1-\gamma) \sum_{t=1}^{\infty} \gamma^{t-1} \mathbf{x}_{t} \mathbf{x}_{t}^{\top}\right] .
\end{array}\right.
$$

Proof. The proof is presented in Appendix I

In this paper, we consider two gradient-based methods.

- Policy gradient descent:

$$
\boldsymbol{\theta}_{k+1}=\boldsymbol{\theta}_{k}-\eta \operatorname{diag}\left(\nabla_{\theta} J\left(\boldsymbol{\theta}_{k}\right), \nabla_{\bar{\theta}} J\left(\boldsymbol{\theta}_{k}\right)\right) .
$$

\section{- Natural policy gradient descent:}

$$
\boldsymbol{\theta}_{k+1}=\boldsymbol{\theta}_{k}-\eta \operatorname{diag}\left(\nabla_{\theta} J\left(\boldsymbol{\theta}_{k}\right), \nabla_{\bar{\theta}} J\left(\boldsymbol{\theta}_{k}\right)\right) \boldsymbol{\Sigma}_{\boldsymbol{\theta}}^{-1} .
$$

To prove our convergence results, we impose extra standard assumptions, described below.

Assumption 4. The initial policy is stable. A policy $\boldsymbol{\theta}$ is said to be stable if $\rho(\mathbf{A}-\mathbf{B} \boldsymbol{\theta})<1$.

Assumption 5. Given the learner, $\mathbb{E}\left[\mathbf{x}_{1}\left(\mathbf{x}_{1}\right)^{\top}\right]$ is positive definite. For the special case of i.i.d. initial states, $\mathbb{E}\left[\mathbf{x}_{1}^{i}\left(\mathbf{x}_{1}^{i}\right)^{\top}\right]=\operatorname{diag}\left(\left(1-\frac{1}{n}\right) \operatorname{cov}\left(x_{1}\right), \frac{1}{n} \operatorname{cov}\left(x_{1}\right)+\right.$ $\left.\mathbb{E}\left[x_{1}\right] \mathbb{E}\left[x_{1}\right]^{\top}\right)$ is positive definite if $\operatorname{cov}\left(x_{1}^{i}\right)=: \operatorname{cov}\left(x_{1}\right)$ and $\mathbb{E}\left[x_{1}^{i}\right] \mathbb{E}\left[x_{1}^{i}\right]^{\top}=: \mathbb{E}\left[x_{1}\right] \mathbb{E}\left[x_{1}\right]^{\top}, i \in \mathbb{N}_{n}$, are positive definite.

Assumption 6. For finite-population model, $\mathbf{Q}$ and $\mathbf{R}$ are positive definite matrices. For the infinite-population case satisfying Assumption 3. $Q$ and $Q+S$ are positive definite.

Assumptions 46 are standard conditions in the literature of LQ reinforcement learning [1], [20], which ensure that for any stable $\boldsymbol{\theta}, J(\boldsymbol{\theta})$ is properly bounded and $\boldsymbol{\Sigma}_{\boldsymbol{\theta}} \succcurlyeq$ $\mathbb{E}\left[\mathbf{x}_{1}\left(\mathbf{x}_{1}\right)^{\top}\right]$ is positive definite. We now show that the bestresponse optimization at the learner satisfies the PolyakLojasiewicz (PL) condition [21], [22], which is a relaxation of the notion of strong convexity. Let $\mu:=\sigma_{\min }\left(\mathbb{E}\left[\mathbf{x}_{1} \mathbf{x}_{1}^{\top}\right]\right)$.

Lemma 2 (PL condition). Let Assumptions 1, 4, 5 and 6 hold. Let also $\boldsymbol{\theta}^{*}$ be the Nash policy in Theorem 1 There exists a positive constant $L_{1}\left(\boldsymbol{\theta}^{*}\right)$ such that

$$
J(\boldsymbol{\theta})-J\left(\boldsymbol{\theta}^{*}\right) \leq L_{1}\left(\boldsymbol{\theta}^{*}\right)\left\|\left[\nabla_{\theta} J(\boldsymbol{\theta}), \nabla_{\bar{\theta}} J(\boldsymbol{\theta})\right]\right\|_{F}^{2},
$$

where $L_{1}\left(\boldsymbol{\theta}^{*}\right)=\frac{n^{2}\left\|\boldsymbol{\Sigma}_{\boldsymbol{\theta}^{*}}\right\|}{4 \mu^{2} \sigma_{\min }(\mathbf{R})}$. For the special case of infinite population (i.e. $n=\infty$ ) with i.i.d. initial states under Assumptions 3, 4, 5, and 6, one has $L_{1}\left(\boldsymbol{\theta}^{*}\right)=$ $\frac{\left\|\boldsymbol{\Sigma}_{\boldsymbol{\theta}^{*}}^{1,1}\right\|}{4 \sigma_{\min }\left(\operatorname{cov}\left(x_{1}\right)\right)^{2} \sigma_{\min }(R)}+\frac{\left\|\boldsymbol{\Sigma}_{\boldsymbol{\theta}^{*}}^{2,2}\right\|}{4 \sigma_{\min }\left(\mathbb{E}\left[x_{1}\right] \mathbb{E}\left[x_{1}\right]^{\top}\right)^{2} \sigma_{\min }\left(R+S^{u}\right)}$.

Proof. The proof is presented in Appendix II.

In the following lemmas, we show that the cost function and its gradient are locally Lipschitz functions.

Lemma 3 (Locally Lipschitz cost function). For any $\boldsymbol{\theta}^{\prime}$ satisfying the inequality $\left\|\boldsymbol{\theta}^{\prime}-\boldsymbol{\theta}\right\|_{F}<\varepsilon(\boldsymbol{\theta})$, there exists a positive constant $L_{2}(\boldsymbol{\theta})$ such that $\left|J\left(\boldsymbol{\theta}^{\prime}\right)-J(\boldsymbol{\theta})\right| \leq L_{2}(\boldsymbol{\theta})\left\|\boldsymbol{\theta}^{\prime}-\boldsymbol{\theta}\right\|_{F}$, where the explicit expressions of $\varepsilon(\boldsymbol{\theta})$ and $L_{2}(\boldsymbol{\theta})$ can be obtained in a similar manner as [20, Lemma 15].

Proof. The proof is omitted due to space limitation.
Lemma 4 (Locally Lipschitz gradient). For any $\boldsymbol{\theta}^{\prime}$ satisfying the inequality $\left\|\boldsymbol{\theta}^{\prime}-\boldsymbol{\theta}\right\|_{F}<\varepsilon(\boldsymbol{\theta})$, there exists a positive constant $L_{3}(\boldsymbol{\theta})$ such that

$$
\left\|\left[\nabla J_{\theta}\left(\boldsymbol{\theta}^{\prime}\right), \nabla J_{\bar{\theta}}\left(\boldsymbol{\theta}^{\prime}\right)\right]-\left[\nabla J_{\theta}(\boldsymbol{\theta}), \nabla J_{\bar{\theta}}(\boldsymbol{\theta})\right]\right\|_{F} \leq L_{3}(\boldsymbol{\theta})\left\|\boldsymbol{\theta}^{\prime}-\boldsymbol{\theta}\right\|_{F},
$$

where the explicit expressions of $\varepsilon(\boldsymbol{\theta})$ and $L_{3}(\boldsymbol{\theta})$ can be obtained in a similar manner as [20, Lemma 16].

Proof. The proof is omitted due to space limitation.

Theorem 2 (Global convergence via model-based gradient). Let Assumptions 5, 4, 5, 5, and 6 hold. For a sufficiently small fixed step size $\eta$ chosen as $\eta=\operatorname{poly}\left(\frac{\mu \sigma_{\min }(\mathbf{Q})}{J\left(\boldsymbol{\theta}_{1}\right)}, \frac{1}{\sqrt{\gamma}\|\mathbf{A}\|}, \frac{1}{\sqrt{\gamma}\|\mathbf{B}\|}, \frac{1}{\|\mathbf{R}\|}, \sigma_{\min }(\mathbf{R})\right)$, and for a sufficiently large number of iterations $K$ such that $K \geq$ $\frac{\left\|\boldsymbol{\Sigma}_{\boldsymbol{\theta}^{*}}\right\|}{\mu} \log \frac{J\left(\boldsymbol{\theta}_{1}\right)-J\left(\boldsymbol{\theta}^{*}\right)}{\varepsilon} \operatorname{poly}\left(\frac{J\left(\boldsymbol{\theta}_{1}\right)}{\mu \sigma_{\min }(\mathbf{Q})}, \sqrt{\gamma}\|\mathbf{A}\|, \sqrt{\gamma}\|\mathbf{B}\|,\|\mathbf{R}\|\right.$, $\left.\frac{1}{\sigma_{\min }(\mathbf{R})}\right)$, the gradient descent algorithm (8) leads to the following bound: $J\left(\boldsymbol{\theta}_{K}\right)-J\left(\boldsymbol{\theta}^{*}\right) \leq \varepsilon$. In particular, for a fixed step size $\eta=\frac{1}{\left\|\mathbf{P}_{n}^{\top} \mathbf{P}_{n}\right\|\left(\|\mathbf{R}\|+\frac{\gamma\|\mathbf{B}\|^{2} J\left(\boldsymbol{\theta}_{1}\right)}{\mu}\right)}$ and for a sufficiently large number of iterations $K$, i.e., $K \geq \frac{\left\|\boldsymbol{\Sigma}_{\boldsymbol{\theta}^{*}}\right\|\left\|\mathbf{P}_{n}^{\top} \mathbf{P}_{n}\right\|}{\mu}\left(\frac{\|\mathbf{R}\|}{\sigma_{\min }(\mathbf{R})}+\frac{\gamma\|\mathbf{B}\|^{2} J\left(\boldsymbol{\theta}_{1}\right)}{\mu \sigma_{\min }(\mathbf{R})}\right) \log \frac{J\left(\boldsymbol{\theta}_{1}\right)-J\left(\boldsymbol{\theta}^{*}\right)}{\varepsilon}$, the natural policy gradient descent algorithm (9) enjoys the bound: $J\left(\boldsymbol{\theta}_{K}\right)-J\left(\boldsymbol{\theta}^{*}\right) \leq \varepsilon$.

Proof. Following the proof technique in [1, Theorem 7], we choose a sufficiently small step size $\eta$ such that the value of the cost decreases at each iteration. More precisely, for the natural policy gradient descent at iteration $K, J\left(\boldsymbol{\theta}_{K+1}\right)-$ $J\left(\boldsymbol{\theta}^{*}\right) \leq\left(1-\frac{\mu \sigma_{\min }(\mathbf{R})}{\left\|\mathbf{P}_{n}^{\top} \mathbf{P}_{n}\right\|\left(\|\mathbf{R}\|+\frac{\gamma\|\mathbf{B}\|^{2} J\left(\boldsymbol{\theta}_{\mathbf{1}}\right)}{\mu}\right)\left\|\boldsymbol{\Sigma}_{\boldsymbol{\theta}^{*}}\right\|}\right)\left(J\left(\boldsymbol{\theta}_{K}\right)-\right.$ $\left.J\left(\boldsymbol{\theta}^{*}\right)\right)=\left(1-\eta \frac{\mu \sigma_{\min }(\mathbf{R})}{\| \boldsymbol{\Sigma}_{\boldsymbol{\theta}^{*} \|}}\right)\left(J\left(\boldsymbol{\theta}_{K}\right)^{\mu}-J\left(\boldsymbol{\theta}^{*}\right)\right)$. The above recursion is contractive for the specified $\eta$.

\section{B. Model-free solution using policy optimization}

It is desired now to develop a model-free RL algorithm.

Lemma 5 (Finite-horizon approximation). For any $\boldsymbol{\theta}$ with $\mathrm{fi}$ nite $J(\boldsymbol{\theta})$, define $\tilde{J}_{T}(\boldsymbol{\theta}):=(1-\gamma) \mathbb{E}\left[\sum_{t=1}^{T} \gamma^{t-1} c_{t}\right]$ and $\tilde{\boldsymbol{\Sigma}}_{\boldsymbol{\theta}}=$ $(1-\gamma) \mathbb{E}\left[\sum_{t=1}^{T} \gamma^{t-1} \mathbf{x}_{t} \mathbf{x}_{t}^{\top}\right]$. Let $\varepsilon(T):=\frac{d_{x}(J(\boldsymbol{\theta}))^{2}}{(1-\gamma) T \mu \sigma_{\min }^{2}(\mathbf{Q})}$ and $\bar{\varepsilon}(T):=\varepsilon(T)\left(\|\mathbf{Q}\|+\|\mathbf{R}\|\|\boldsymbol{\theta}\|^{2}\right)$, then $\left\|\tilde{\boldsymbol{\Sigma}}_{\boldsymbol{\theta}}-\boldsymbol{\Sigma}_{\boldsymbol{\theta}}\right\| \leq \varepsilon(T)$ and $\left|\tilde{J}_{T}(\boldsymbol{\theta})-J(\boldsymbol{\theta})\right| \leq \bar{\varepsilon}(T)$.

Proof. The proof is omitted due to space limitation.

Let $\mathbb{S}_{r}$ be a set of uniformly distributed points with norm $r>0$ (e.g., the surface of a sphere). In addition, let $\mathbb{B}_{r}$ denote the set of all uniformly distributed points whose norms are at most $r$ (e.g., all points within the sphere). For a matrix $\tilde{\boldsymbol{\theta}}=$ $\operatorname{diag}(\tilde{\theta}, \tilde{\bar{\theta}})$, these distributions are defined over the Frobenius norm ball. Hence, $J_{r}(\boldsymbol{\theta})=\mathbb{E}_{\tilde{\boldsymbol{\theta}} \sim \mathbb{B}_{r}}[J(\boldsymbol{\theta}+\tilde{\boldsymbol{\theta}})]$. Since the expectation can be expressed as an integral function, one can use Stokes' formula to compute the gradient of $J_{r}(\boldsymbol{\theta})$ with only query access to the function values.

Lemma 6 (Zeroth-order optimization). For a smoothing factor $r>0,\left[\nabla_{\theta} J(\boldsymbol{\theta}), \nabla_{\bar{\theta}} J(\boldsymbol{\theta})\right]=\frac{2 d_{x} d_{u}}{r^{2}} \mathbb{E}_{\tilde{\boldsymbol{\theta}} \sim \mathbb{S}_{r}}[J(\boldsymbol{\theta}+\tilde{\boldsymbol{\theta}})[\tilde{\theta}, \tilde{\bar{\theta}}]]$.

Proof. The proof follows directly from the zeroth-order optimization approach [23, Lemma 1]. 
Lemma 7. Let $\tilde{\boldsymbol{\theta}}_{1}, \ldots, \tilde{\boldsymbol{\theta}}_{L}, L \in \mathbb{N}$, be i.i.d. samples drawn uniformly from $\mathbb{S}_{r}$. There exists $\varepsilon(L):=$ $\operatorname{poly}(1 / L)>0, \quad$ such that $\left[\tilde{\nabla}_{\theta}^{L} J(\boldsymbol{\theta}), \tilde{\nabla}_{\bar{\theta}}^{L} J(\boldsymbol{\theta})\right]=$ $\frac{2 d_{x} d_{u}}{r^{2} L} \sum_{l=1}^{L} J\left(\boldsymbol{\theta}+\tilde{\boldsymbol{\theta}}_{l}\right)[\tilde{\theta}, \tilde{\bar{\theta}}]$ converges to $\left[\nabla_{\theta} J(\boldsymbol{\theta}), \nabla_{\bar{\theta}} J(\boldsymbol{\theta})\right]$ in the Frobenius norm with a probability greater than $1-\left(\frac{2 d_{x} d_{u}}{\varepsilon(L)}\right)^{-2 d_{x} d_{u}}$. From Lemma 5 there exists $\varepsilon(L, T):=$ $\operatorname{poly}(1 / L, 1 / T)>0$ such that $\left[\nabla_{\theta}^{L, T} J(\boldsymbol{\theta}), \tilde{\nabla}_{\bar{\theta}}^{L, T} J(\boldsymbol{\theta})\right]=$ $\frac{2 d_{x} d_{u}(1-\gamma)}{r^{2} L} \sum_{l=1}^{L}\left[\sum_{t=1}^{T} \gamma^{t-1}\left(c_{t}\right)\right]\left[\tilde{\theta}_{l}, \tilde{\bar{\theta}}_{l}\right]$ is $\varepsilon(L, T)$ close to $\left[\nabla_{\theta} J(\boldsymbol{\theta}), \nabla_{\bar{\theta}} J(\boldsymbol{\theta})\right]$ with a probability greater than $1-$ $\left(\frac{2 d_{x} d_{u}}{\varepsilon(L, T)}\right)^{-2 d_{x} d_{u}}$ in the Frobenius norm.

Proof. The proof is omitted due to space limitation.

Theorem 3 (Global convergence via model-free gradient). Let Assumptions 1 4, 5 and 6 hold. For a sufficiently large horizon $T$ and samples $L$, model-free gradient descent and natural policy gradient decent with the empirical gradient in Lemma 7 and covariance matrix in Lemma 5 converge to the model-based solutions in Theorem 2 In particular, the gradient descent algorithm converges with a probability greater

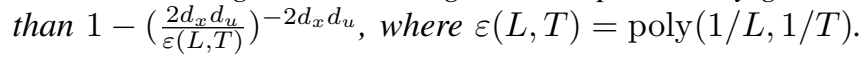

Proof. From [1, Theorem 31] and Theorem 2, one has the following inequality at iteration $K \in \mathbb{N}$ for a sufficiently small step size $\eta \leq \eta_{\max }, J\left(\boldsymbol{\theta}_{K+1}\right)-J\left(\boldsymbol{\theta}^{*}\right) \leq$ $\left(1-\eta \eta_{\text {max }}^{-1}\right)\left(J\left(\boldsymbol{\theta}_{K}\right)-J\left(\boldsymbol{\theta}^{*}\right)\right)$. At iteration $K$, denote by $\tilde{\nabla}_{K}$ the empirical gradient and by $\hat{\boldsymbol{\theta}}_{K+1}=\boldsymbol{\theta}_{K}-\eta \tilde{\nabla}_{K}$ the update with the empirical gradient. From Lemma 3 , $\left|J\left(\hat{\boldsymbol{\theta}}_{K+1}\right)-J\left(\boldsymbol{\theta}_{K+1}\right)\right| \leq \frac{1}{2} \eta \eta_{\max }^{-1} \varepsilon(L, T)$, when $\| \hat{\boldsymbol{\theta}}_{K+1}-$ $\boldsymbol{\theta}_{K+1} \| \leq \frac{1}{2} \eta \eta_{\max }^{-1} \varepsilon(L, T)\left(1 / L_{2}\left(\boldsymbol{\theta}_{K+1}\right)\right)$, upon noting that $\hat{\boldsymbol{\theta}}_{K+1}-\boldsymbol{\theta}_{K+1}=\eta\left(\nabla_{K}-\tilde{\nabla}_{K}\right)$ and $\left\|\nabla_{K}-\tilde{\nabla}_{K}\right\| \leq$ $\frac{1}{2} \eta_{\max }^{-1} \varepsilon(L, T)\left(1 / L_{2}\left(\boldsymbol{\theta}_{K+1}\right)\right)$. According to the Bernstein inequality, the above inequality holds with a probability greater than $1-\left(\frac{2 d_{x} d_{u}}{\varepsilon(L, T)}\right)^{-2 d_{x} d_{u}}$. Therefore, from Lemmas 4 and 7 the distance between the empirical gradient and the exact one monotonically decreases as the number of samples and rollouts increases, provided that the smoothing factor $r$ is sufficiently small. Consequently, one arrives at $J\left(\hat{\boldsymbol{\theta}}_{K+1}\right)-J\left(\boldsymbol{\theta}^{*}\right) \leq\left(1-\frac{1}{2} \eta \eta_{\max }^{-1}\right)\left(J\left(\boldsymbol{\theta}_{K}\right)-J\left(\boldsymbol{\theta}^{*}\right)\right)$, when $J\left(\boldsymbol{\theta}_{K}\right)-J\left(\boldsymbol{\theta}^{*}\right) \leq \varepsilon(L, T)$. This recursion is contractive; i.e., the rest of the proof will be similar to that of Theorem 2

\section{Simulations}

In this section, simulations are conducted to demonstrate the global convergence of the proposed gradient methods. To compute the Nash policy, plotted in dashed lines in the figures, we use the solution of equation (4).

Example 1. Consider a dynamic game with the following parameters: $\eta=0.1, n=100, T=100, L=3, A=$ $0.7, B=0.4, \bar{A}=0, \bar{B}=0, Q=1, R=1, S^{x}=4, S^{u}=$ $0, \bar{Q}=0, \bar{R}=0, \Sigma_{x}=1$ and $\Sigma_{w}=0.4$. It is observed in Figure 1 that natural policy gradient descent reaches the Nash strategy faster than the gradient descent.

Example 2. Let the system parameters be $\eta=0.04, n=$ $10, T=10, r=0.09, L=1500, A=1, B=0.5, \bar{A}=$ $0, \bar{B}=0, Q=1, R=1, S^{x}=2, S^{u}=0, \bar{Q}=1, \bar{R}=$ $0, r=0.09, \Sigma_{x}=0.05$ and $\Sigma_{w}=0.01$. The model-free policy gradient algorithm was run on a $2.7 \mathrm{GHz}$ Intel Core i5
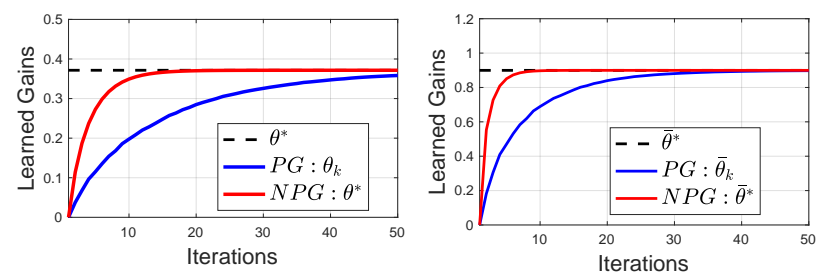

Fig. 1. Convergence of the model-based gradient descent and natural policy gradient descent algorithms in Example 1.

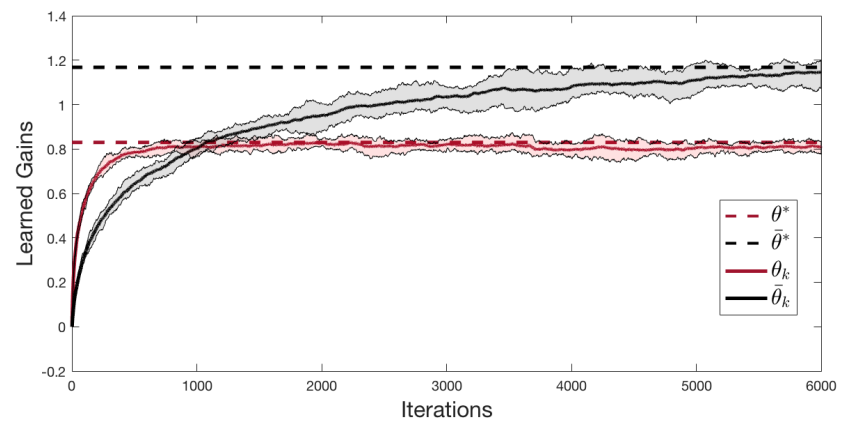

Fig. 2. Convergence of the proposed model-free algorithm in Example 2.
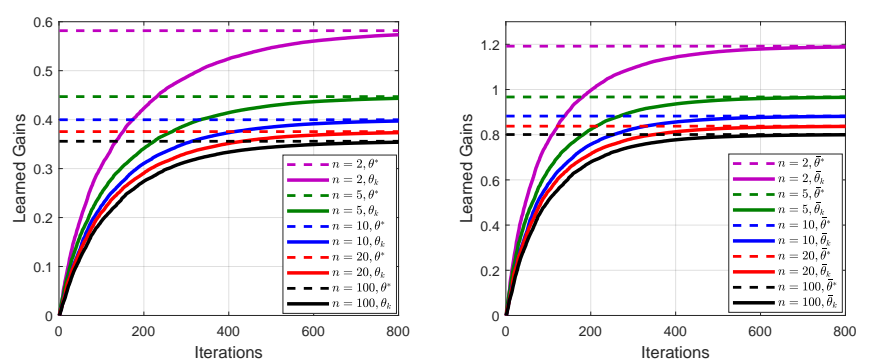

Fig. 3. The effect of the number of players on the policy in Example 3.

processor for 10 random seeds. After 6000 iterations, which took roughly 10 hours, both $\theta$ and $\bar{\theta}$ reached their optimal values as depicted in Figure 2

Example 3. In this example, let the system parameters be $\eta=0.1, T=100, L=3, A=0.8, B=0.2, \bar{A}=$ $0, \bar{B}=0, Q=1, R=1, S^{x}=2, S^{u}=0, \bar{Q}=4$, $\bar{R}=0, \Sigma_{x}=1$ and $\Sigma_{w}=0.1$. To investigate the effect of the number of players, we considered five different values for $n \in\{2,5,10,20,100\}$. It is shown in Figure 3 that the policies converge to a limit as the number of players increases, which is known as the mean-field limit.

\section{CONCLUSIONS}

In this paper, we investigated model-based and modelfree gradient descent and natural policy gradient descent algorithms for LQ deep structured games with homogeneous weights. It was shown theoretically and verified by simulations, that the gradient-based methods enjoy the global convergence to the sequential Nash solution. One of the main features of the proposed solutions is that their planning space is independent of the number of players. The obtained results naturally extend to asymptotically vanishing weights 
and other variants of policy gradient algorithms such as REINFORCE and actor-critic methods.

\section{REFERENCES}

[1] M. Fazel, R. Ge, S. M. Kakade, and M. Mesbahi, "Global convergence of policy gradient methods for the linear quadratic regulator," arXiv preprint arXiv:1801.05039, 2018.

[2] K. Zhang, Z. Yang, and T. Basar, "Policy optimization provably converges to Nash equilibria in zero-sum linear quadratic games," in Advances in NeurIPS, 2019, pp. 11 598-11610.

[3] E. Mazumdar, L. J. Ratliff, M. I. Jordan, and S. S. Sastry, "Policy-gradient algorithms have no guarantees of convergence in continuous action and state multi-agent settings," arXiv preprint arXiv:1907.03712, 2019.

[4] J. Arabneydi and A. G. Aghdam, "Deep teams: Decentralized decision making with finite and infinite number of agents," IEEE Transactions on Automatic Control, DOI: 10.1109/TAC.2020.2966035, 2020.

[5] J. Arabneydi, A. G. Aghdam, and R. P. Malhamé, "Explicit sequential equilibria in LQ deep structured games and weighted mean-field games," conditionally accepted in Automatica, 2020.

[6] J. Arabneydi and A. G. Aghdam, "Deep structured teams with linear quadratic model: Partial equivariance and gauge transformation," [Online]. Available at https://arxiv.org/abs/1912.03951, 2019.

[7] — "Deep structured teams and games with Markov-chain model: Finite and infinite number of players," Submitted, 2019.

[8] J. Arabneydi, M. Roudneshin, and A. G. Aghdam, "Reinforcement learning in deep structured teams: Initial results with finite and infinite valued features," in Proceedings of IEEE Conference on Control Technology and Applications, 2020.

[9] V. Fathi, J. Arabneydi, and A. G. Aghdam, "Reinforcement learning in linear quadratic deep structured teams: Global convergence of policy gradient methods," in Proceedings of the 59th IEEE Conference on Decision and Control, 2020.

[10] P. E. Caines, M. Huang, and R. P. Malhamé, "Mean field games," in Handbook of Dynamic Game Theory, T. Başar and G. Zaccour, Eds. Springer International Publishing, 2018, pp. 345-372.

[11] J. Arabneydi, "New concepts in team theory: Mean field teams and reinforcement learning," Ph.D. dissertation, Department of Electrical and Computer Engineering, McGill University, Canada, 2016.

[12] R. Elliott, X. Li, and Y.-H. Ni, "Discrete time mean-field stochastic linear-quadratic optimal control problems," Automatica, vol. 49, no. 11, pp. 3222-3233, 2013.

[13] A. Bensoussan, J. Frehse, and P. Yam, Mean field games and mean field type control theory. Springer-Verlag New York, 2013.

[14] R. Carmona and F. Delarue, Probabilistic Theory of Mean Field Games with Applications I-II. Springer, 2018.

[15] J. Arabneydi and A. Mahajan, "Linear quadratic mean field teams: Optimal and approximately optimal decentralized solutions," Available at https://arxiv.org/abs/1609.00056, 2016.

[16] - , "Team-optimal solution of finite number of mean-field coupled LQG subsystems," in Proceedings of the 54th IEEE Conference on Decision and Control, 2015, pp. 5308 - 5313.

[17] M. Baharloo, J. Arabneydi, and A. G. Aghdam, "Near-optimal control strategy in leader-follower networks: A case study for linear quadratic mean-field teams," in Proceedings of the 57th IEEE Conference on Decision and Control, 2018, pp. 3288-3293.

[18] — "Minmax mean-field team approach for a leader-follower network: A saddle-point strategy," IEEE Control Systems Letters, vol. 4, no. 1, pp. 121-126, 2019.

[19] J. Arabneydi and A. G. Aghdam, "Optimal dynamic pricing for binary demands in smart grids: A fair and privacy-preserving strategy," in Proceedings of American Control Conference, 2018, pp. 5368-5373.

[20] D. Malik, A. Pananjady, K. Bhatia, K. Khamaru, P. L. Bartlett, and M. J. Wainwright, "Derivative-free methods for policy optimization: Guarantees for linear quadratic systems," Journal of Machine Learning Research, vol. 21, no. 21, pp. 1-51, 2020.

[21] B. Polyak, "Gradient methods for solving equations and inequalities," USSR Computational Mathematics and Mathematical Physics, vol. 4, no. 6, pp. 17-32, 1964.

[22] S. Lojasiewicz, "A topological property of real analytic subsets," Coll. du CNRS, Les equations aux d'eriv'ees partielles, pp. 87-89, 1963.

[23] A. D. Flaxman, A. T. Kalai, and H. B. McMahan, "Online convex optimization in the bandit setting: Gradient descent without a gradient," in Proceedings of the ACM-SIAM Symposium on Discrete Algorithms. Society for Industrial and Applied Mathematics, 2005, p. 385-394.

\section{APPENDIX I}

PROOF OF LEMMA 1

To compute the best-response of the learner, we fix the strategies of other players, and then find the gradient of the cost function with respect to $\theta$ and $\bar{\theta}$. Suppose player $i \in \mathbb{N}_{n}$ uses the strategy $u_{t}^{i}=\theta^{i} x_{t}^{i}+\left(\bar{\theta}^{i}-\theta^{i}\right) \bar{x}_{t}$. Therefore, one has:

$\mathbf{u}_{t}^{i}=\left[\begin{array}{cc}\left(1-\frac{1}{n}\right) \theta^{i} & \left(1-\frac{1}{n}\right) \bar{\theta}^{i} \\ \frac{1}{n} \theta^{i} & \frac{1}{n} \theta^{i}\end{array}\right] \mathbf{x}_{t}^{i}+\sum_{j \neq i}\left[\begin{array}{cc}-\frac{1}{n} \theta^{j} & -\frac{1}{n} \bar{\theta}^{j} \\ \frac{1}{n} \theta^{j} & \frac{1}{n} \bar{\theta}^{j}\end{array}\right] \mathbf{x}_{t}^{j}$

From (2) and (3), $J_{\mathbf{x}_{1}^{i}}(\boldsymbol{\theta})=\mathbb{E}\left[\left(\mathbf{x}_{1}^{i}\right)^{\top} \mathbf{Q} \mathbf{x}_{1}^{i}+\left(\mathbf{u}_{1}^{i}\right)^{\top} \mathbf{R} \mathbf{u}_{1}^{i}\right]+$ $\gamma J_{\mathbf{x}_{2}^{i}}(\boldsymbol{\theta})=\mathbb{E}\left[\left(\mathbf{x}_{1}^{i}\right)^{\top} \mathbf{Q} \mathbf{x}_{1}^{i}+\left(\mathbf{u}_{1}^{i}\right)^{\top} \mathbf{R} \mathbf{u}_{1}^{i}\right]+\gamma \mathbb{E}\left[\left(\mathbf{x}_{2}^{i}\right)^{\top} \mathbf{M}(\boldsymbol{\theta}) \mathbf{x}_{2}^{i}\right]$. Taking the derivatives with respect to $\theta^{i}$ and $\bar{\theta}^{i}$, and then making $\theta^{i}=\theta^{j}=\theta$ and $\bar{\theta}^{i}=\bar{\theta}^{j}=\bar{\theta}$, leads to:

$$
\left\{\begin{array}{l}
\nabla_{\theta} J_{\mathbf{x}_{1}}(\boldsymbol{\theta})=2\left(\left(1-\frac{1}{n}\right)\left(\mathbf{R}^{1,1}+\gamma \mathbf{B}^{1,1} \mathbf{T}^{\boldsymbol{T}} \mathbf{M}^{1,1}(\boldsymbol{\theta}) \mathbf{B}^{1,1}\right)\right. \\
\left.+\frac{1}{n}\left(\mathbf{R}^{2,1}+\gamma \mathbf{B}^{2,1} \mathbf{M}^{2,1}(\boldsymbol{\theta}) \mathbf{B}^{2,1}\right)\right) \theta \mathbb{E}\left[\Delta x_{1} \Delta x_{1}^{\boldsymbol{\top}}\right] \\
+2\left(\left(1-\frac{1}{n}\right)\left(\mathbf{R}^{1,2}+\gamma \mathbf{B}^{1,2 \boldsymbol{\top}} \mathbf{M}^{1,2}(\boldsymbol{\theta}) \mathbf{B}^{1,2}\right)+\frac{1}{n}\left(\mathbf{R}^{2,2}\right.\right. \\
\left.\left.+\gamma \mathbf{B}^{2,2 \boldsymbol{\top}} \mathbf{M}^{2,2}(\boldsymbol{\theta}) \mathbf{B}^{2,2}\right)\right) \bar{\theta} \mathbb{E}\left[\bar{x}_{1} \Delta x_{1}^{\boldsymbol{\top}}\right]+\gamma \nabla_{\theta} J_{\mathbf{x}_{2}}(\boldsymbol{\theta}), \\
\nabla_{\bar{\theta}} J_{\mathbf{x}_{1}}(\boldsymbol{\theta})=2\left(\left(1-\frac{1}{n}\right)\left(\mathbf{R}^{1,1}+\gamma \mathbf{B}^{1,1} \mathbf{M}^{\top} \mathbf{M}^{1,1}(\boldsymbol{\theta}) \mathbf{B}^{1,1}\right)\right. \\
\left.+\frac{1}{n}\left(\mathbf{R}^{2,1}+\gamma \mathbf{B}^{2,1} \mathbf{M}^{2,1}(\boldsymbol{\theta}) \mathbf{B}^{2,1}\right)\right) \theta \mathbb{E}\left[\Delta x_{1} \bar{x}_{1}^{\boldsymbol{\top}}\right] \\
+2\left(\left(1-\frac{1}{n}\right)\left(\mathbf{R}^{1,2}+\gamma \mathbf{B}^{1,2} \mathbf{M}^{1,2}(\boldsymbol{\theta}) \mathbf{B}^{1,2}\right)\right. \\
\left.+\frac{1}{n}\left(\mathbf{R}^{2,2}+\gamma \mathbf{B}^{2,2 \boldsymbol{\top}} \mathbf{M}^{2,2}(\boldsymbol{\theta}) \mathbf{B}^{2,2}\right)\right) \bar{\theta} \mathbb{E}\left[\bar{x}_{1} \bar{x}_{1}^{\top}\right]+\gamma \nabla_{\bar{\theta}} J_{\mathbf{x}_{2}}(\boldsymbol{\theta}) .
\end{array}\right.
$$

The the rest of the proof follows from the recursive application of (10) and equations (4), (5) and (7).

\section{APPENDIX II}

PROOF OF LEMMA 2

Let $\tilde{\mathbf{P}}_{n}:=\operatorname{diag}\left(\left(1-\frac{1}{n}\right) \mathbf{I}_{d_{u} \times d_{u}}, \frac{1}{n} \mathbf{I}_{d_{u} \times d_{u}}\right)$. We express (6) in terms of the square matrix $\tilde{\mathbf{P}}_{n}$ such that $\Delta J_{\theta}(\boldsymbol{\theta})=$ : $\Delta J_{\theta}^{1}(\boldsymbol{\theta})+\Delta J_{\theta}^{2}(\boldsymbol{\theta})$ and $\Delta J_{\bar{\theta}}(\boldsymbol{\theta})=: \Delta J_{\bar{\theta}}^{1}(\boldsymbol{\theta})+\Delta J_{\bar{\theta}}^{2}(\boldsymbol{\theta})$, where

$$
\nabla_{\boldsymbol{\theta}} \tilde{J}:=\left[\begin{array}{cc}
\Delta J_{\theta}^{1}(\boldsymbol{\theta}) & \Delta J_{\bar{\theta}}(\boldsymbol{\theta}) \\
\Delta J_{\theta}^{2}(\boldsymbol{\theta}) & \Delta J_{\bar{\theta}}(\boldsymbol{\theta})
\end{array}\right]=2 \tilde{\mathbf{P}}_{n} \mathbf{E}_{\boldsymbol{\theta}} \boldsymbol{\Sigma}_{\boldsymbol{\theta}} .
$$

Following [1, Lemma 10] and after some algebraic manipulations, we can derive the following inequality for sequences $\left\{\mathbf{x}_{t}^{*}\right\}_{t=1}^{\infty}$ and $\left\{\mathbf{u}_{t}^{*}\right\}_{t=1}^{\infty}$ generated by the Nash policy $\boldsymbol{\theta}^{*}$. In particular, from (6), $\Sigma_{\boldsymbol{\theta}} \succcurlyeq$ $\mathbb{E}\left[\left(\mathbf{x}_{1} \mathbf{x}_{1}^{\top}\right)\right]$, and the fact that $\tilde{\mathbf{P}}_{n}$ is positive definite for any finite $n$, it results that: $J(\boldsymbol{\theta})-J\left(\boldsymbol{\theta}^{*}\right) \leq$ $(1-\gamma) \mathbb{E} \sum_{t=1}^{\infty} \gamma^{t-1} \operatorname{Tr}\left(\mathbf{x}_{t}^{*} \mathbf{x}_{t}^{* \top} \mathbf{E}_{\boldsymbol{\theta}}^{\boldsymbol{\top}}\left(\mathbf{R}+\gamma \mathbf{B}^{\top} \mathbf{M}_{\boldsymbol{\theta}} \mathbf{B}\right)^{-1} \mathbf{E}_{\boldsymbol{\theta}}\right)=$ $\operatorname{Tr}\left(\Sigma_{\boldsymbol{\theta}^{*}} \mathbf{E}_{\boldsymbol{\theta}}^{\boldsymbol{\top}}\left(\mathbf{R}+\gamma \mathbf{B}^{\top} \mathbf{M}_{\boldsymbol{\theta}} \mathbf{B}\right)^{-1} \mathbf{E}_{\boldsymbol{\theta}}\right) \leq \frac{\left\|\boldsymbol{\Sigma}_{\boldsymbol{\theta}}\right\|}{\sigma_{\min }(\mathbf{R})} \operatorname{Tr}\left(\mathbf{E}_{\boldsymbol{\theta}}^{\boldsymbol{\top}} \mathbf{E}_{\boldsymbol{\theta}}\right)=$ $\frac{\left\|\boldsymbol{\Sigma}_{\boldsymbol{\theta}^{*}}\right\|}{4 \sigma_{\min }(\mathbf{R})} \operatorname{Tr}\left(\boldsymbol{\Sigma}_{\boldsymbol{\theta}}{ }^{-1} \nabla_{\boldsymbol{\theta}} \tilde{J}^{\top} \mathbf{P}_{n}^{-2} \nabla_{\boldsymbol{\theta}} \tilde{J} \boldsymbol{\Sigma}_{\boldsymbol{\theta}}{ }^{-1}\right) \leq \frac{0.25 \mu^{-2}\left\|\boldsymbol{\Sigma}_{\boldsymbol{\theta}}\right\|}{\sigma_{\min }\left(\tilde{\mathbf{P}}_{n}\right)^{2} \sigma_{\min }(\mathbf{R})}$ $\left\|\left[\nabla_{\theta} J(\boldsymbol{\theta}), \nabla_{\bar{\theta}} J(\boldsymbol{\theta})\right]\right\|_{F}^{2}$. For $n=\infty, \tilde{\mathbf{P}}_{n}$ is not invertible; however, equation (4) under Assumption 3 decomposes into two decoupled standard Riccati equations with matrices $(A, B, Q, R)$ and $\left(A, B, Q+S^{x}, R+S^{u}\right)$. By following the approach proposed in [1, Lemma 11], it is straightforward to show that the cost difference in this case is upper bounded by: $\frac{\left\|\boldsymbol{\Sigma}_{\boldsymbol{\theta}^{*}}^{1,1}\right\|}{4 \sigma_{\min }\left(\operatorname{cov}\left(x_{1}\right)\right)^{2} \sigma_{\min }(R)}\left\|\nabla_{\theta} J(\boldsymbol{\theta})\right\|_{F}^{2}+$ $\frac{\left\|\boldsymbol{\Sigma}_{\boldsymbol{\theta}^{*}}^{2,2}\right\|}{4 \sigma_{\min }\left(\mathbb{E}\left[x_{1}\right] \mathbb{E}\left[x_{1}\right]^{\top}\right)^{2} \sigma_{\min }\left(R+S^{u}\right)}\left\|\nabla_{\bar{\theta}} J(\boldsymbol{\theta})\right\|_{F}^{2}$. 